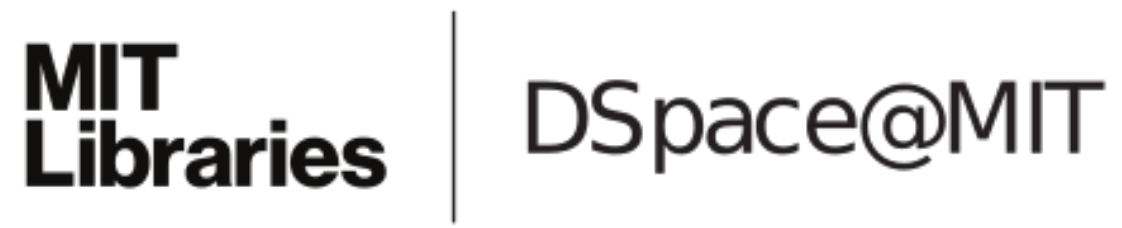

\author{
MIT Open Access Articles
}

Layer-specific interhemispheric functional connectivity in the somatosensory cortex of rats: resting state electrophysiology and $\mathrm{fMRI}$ studies

The MIT Faculty has made this article openly available. Please share how this access benefits you. Your story matters.

Citation: Baek, Kwangyeol et al. "Layer-Specific Interhemispheric Functional Connectivity in the Somatosensory Cortex of Rats: Resting State Electrophysiology and fMRI Studies." Brain Structure and Function 221.5 (2016): 2801-2815.

As Published: http://dx.doi.org/10.1007/s00429-015-1073-0

Publisher: Springer Berlin Heidelberg

Persistent URL: http://hdl.handle.net/1721.1/106537

Version: Author's final manuscript: final author's manuscript post peer review, without publisher's formatting or copy editing

Terms of Use: Article is made available in accordance with the publisher's policy and may be subject to US copyright law. Please refer to the publisher's site for terms of use. 


\title{
Layer-specific interhemispheric functional connectivity in the somatosensory cortex of rats: resting state electrophysiology and fMRI studies
}

\author{
Kwangyeol Baek ${ }^{1,2}$ - Woo Hyun Shim ${ }^{1,2}$ - Jaeseung Jeong ${ }^{1}$ - Harsha Radhakrishnan ${ }^{3}$. \\ Bruce R. Rosen ${ }^{2,4}$ - David Boas ${ }^{2,4}$ - Maria Franceschini ${ }^{2,4} \cdot$ Bharat B. Biswal $^{5}$. \\ Young R. Kim ${ }^{2,4,6}$
}

Received: 29 June 2014/ Accepted: 1 June 2015/Published online: 16 June 2015

(C) Springer-Verlag Berlin Heidelberg 2015

\begin{abstract}
The spontaneous cerebral hemodynamic fluctuations observed during the resting state have been frequently visualized using functional magnetic resonance imaging (rsfMRI). However, the neuronal populations and neuroelectric characteristics underlying the functional connectivity of cerebrohemodynamic activities are poorly understood. We investigated the characteristics of bihemispheric functional connectivity via electrophysiology and rsfMRI in the primary sensory cortex of rats anesthetized by $\alpha$-chloralose. Unlike the evoked responses, the spontaneous electrophysiological activity was concentrated
\end{abstract}

K. Baek and W. H. Shim contributed equally to this study.

Electronic supplementary material The online version of this article (doi:10.1007/s00429-015-1073-0) contains supplementary material, which is available to authorized users.

Jaeseung Jeong

jsjeong@kaist.ac.kr

$\bowtie$ Young R. Kim

spmn@nmr.mgh.harvard.edu

1 Department of Bio and Brain Engineering, Korea Advanced Institute of Science and Technology (KAIST),

Daejeon 305-701, South Korea

2 Athinoula A. Martinos Center for Biomedical Imaging, Massachusetts General Hospital, 149 13th St., Charlestown, MA 02129, USA

3 Center for Neural Engineering, Pennsylvania State University, University Park, PA, USA

4 Harvard Medical School, Boston, MA, USA

5 Department of Biomedical Engineering, New Jersey Institute of Technology, Newark, NJ, USA

6 Ulsan National Institute of Science and Technology, Ulsan, South Korea in the infragranular layers and could be classified into subtypes with distinctive current sources and sinks. Both neuroelectric and rsfMRI signals were interhemispherically correlated in a layer-specific manner, suggesting that there are independent neural inputs to infragranular and granular/supragranular layers. The majority of spontaneous electrophysiological activities were bilaterally paired with delays of up to $\sim 50 \mathrm{~ms}$ between each pair. The variable interhemispheric delay implies the involvement of indirect, multi-neural pathways. Our findings demonstrated the diverse activity patterns of layer-specific electrophysiological substrates and suggest the recruitment of multiple, non-specific brain regions in construction of interhemispheric functional connectivity.

Keywords Interhemispheric connectivity - Spontaneous activity $\cdot$ Resting state fMRI $\cdot$ Local field potential · Cortical layers

\section{Introduction}

The brain exhibits abundant spontaneous activity even in the absence of external sensory input or behavioral activity. Spontaneous neural activity is estimated to consume up to $80 \%$ of the total energy in the resting brain (Raichle and Mintun 2006; Shulman et al. 2004), whereas sensory stimulation increases the regional metabolic expenditure by only a small fraction $(\sim 20 \%)$. Despite the significant energy consumption, only marginal attention had been devoted to understanding the role of spontaneous neural activity until Biswal and his colleagues showed significant temporal correlations among the low-frequency components of spontaneous blood oxygen level dependent (BOLD) fluctuations in the sensorimotor system of the 
resting human brain (Biswal et al. 1995). The dynamic correlation of spontaneous neural activities was suggested between functionally related brain regions, and the analysis of the resting state neural signals has become widely accepted for mapping the functional connectivity (Friston 1994). Among a number of techniques designed for acquiring spatiotemporal neural signals, the use of the BOLD resting state functional magnetic resonance imaging (rsfMRI) method has been highly popular due to excellent spatial resolution, which reveals distinct groups of functional networks, including somatomotor, visual, auditory, task-negative, hippocampal, language-related, and attentional neural networks (Fox and Raichle 2007). Although highly suggestive of neural connections, the coupling between the spontaneous neural activity and hemodynamic rsfMRI signals is still not fully established, which underpins the basis of the rsfMRI strategy for identifying the functional connectivity. In fact, the rsfMRI signal can be significantly affected by cardiovascular activity alone, devoid of neural correlates (Shmueli et al. 2007; Kiviniemi et al. 2003). Acknowledging such limitations, the goal of the current study was to reveal and characterize the neural populations and involved spontaneous neuroelectric activity and the subsequent rsfMRI BOLD fluctuations that give rise to connective networks across bilateral hemispheres in the anesthetized rat brain.

To date, studies of the neural correlates involved with spontaneous BOLD activity have used electrophysiological recording methods such as the electroencephalography (EEG), electrocorticography (ECoG), local field potential (LFP), and multi-unit activity (MUA) (Leopold and Maier 2012). Leopold and his colleagues have demonstrated that spontaneous BOLD fluctuations correlate with slow modulation of the spiking rate, MUA power, and LFP power (gamma band and $2-15 \mathrm{~Hz}$ range) in the monkey visual cortex at rest (Shmuel and Leopold 2008; Scholvinck et al. 2010). In human subjects, slow modulation of the firing rate and gamma LFP power was found to be bilaterally synchronized between auditory cortices, and interhemispheric correlation was also demonstrated in gamma ECoG power change in sensory cortices including visual system (Nir et al. 2008). In addition, delta oscillations in EEG recordings $(0-4 \mathrm{~Hz})$ were bilaterally synchronized in the primary somatosensory cortices of anesthetized rats, which supports the interhemispheric correlation of spontaneous BOLD fluctuations ( $\mathrm{Lu}$ et al. 2007). These data provide a tentative description of the neural basis for spontaneous activity. However, a detailed understanding of the neuronal populations and the characteristics of spontaneous neuroelectric events, which induce correlative resting state hemodynamic fluctuations, remains to be elucidated. In the current study, the electrophysiological basis of functional connectivity was explored in the cortical laminar structures, and we focused on both spontaneous electrophysiological activity and the resulting rsfMRI signals.

The neocortex in mammals is characterized by a welldeveloped laminar architecture and anatomical connections to other brain regions. In particular, the well-known afferent thalamic pathways to the neocortex have provided a key for identifying the laminar populations related to stimulus-evoked activities (e.g., electrical forelimb stimulation) and its interactions with other neural centers. With this in mind, we examined the spatiotemporal traits of both spontaneous and evoked activities across bilateral primary somatosensory cortices of rats using a linear electrode array with multiple electrical contacts. The structural origin and laminar specificity of the spontaneous activity, which accounted for the interhemispherically synchronous neural signals, were examined using a pair of laminar electrodes spanning the whole cortical depth. Additionally, to explore a possible link to the neurovascular function, the features of interhemispheric neural connectivity derived from laminar recordings were compared with the independent high-resolution rsfMRI results. In the present study, we hypothesized that neuronal population underlying spontaneous activity is distinct from one underlying evoked activity so that spontaneous activity should be distinct in spatiotemporal properties and relatively independent from evoked activity. We also expected that only spontaneous activity is synchronized between bilateral neocortices, particularly with layer-specific correlation.

\section{Materials and methods}

\section{Animal preparation}

Male Sprague-Dawley rats weighing 300-350 g were used in the electrophysiology and the rsfMRI experiment $(n=6$ for electrophysiology, $n=5$ for rsfMRI). All experimental procedures were approved by the Massachusetts General Hospital Subcommittee on Research Animal Care. The rats were initially anesthetized with $2.0 \%$ isoflurane in $\mathrm{O}_{2}$ for $3 \mathrm{~min}$, and were maintained with $1.5 \%$ isoflurane in a mixture of air and oxygen during the surgical preparation. A polyethylene catheter (PE-50) was used to cannulate the right femoral artery and vein, enabling blood pressure monitoring, blood gas analysis, and anesthetic administration. Thereafter, the animals were tracheotomized and mechanically ventilated. For the animals subjected to electrophysiology, the areas of skull and dura mater overlying the bilateral primary somatosensory cortices (S1) were removed for electrophysiological recordings.

The isoflurane was discontinued prior to electrophysiological recordings and rsfMRI, and the anesthesia was switched to a $50 \mathrm{mg} / \mathrm{kg}$ intravenous bolus of $\alpha$-chloralose 
followed by continuous intravenous infusion at $40 \mathrm{mg} / \mathrm{kg} /$ h. All electrophysiological recordings were performed under $\alpha$-chloralose anesthesia. The body temperature $\left(37.0^{\circ} \mathrm{C}\right)$ was maintained with a temperature-controlled heating pad placed under the rat's torso and was monitored with a rectal probe. The mean arterial blood pressure, heart rate, arterial blood gas, and body temperature were monitored and carefully maintained at normal levels throughout the experiment. The duration of surgical preparation was approximately 2-3 h. The duration of in vivo electrophysiology recordings was approximately $3 \mathrm{~h}$. The same preparatory procedure was performed for rsfMRI, except for the removal of skull and dura mater.

\section{Electrophysiological recordings}

The electrophysiological recordings were performed in the forelimb region of the bilateral primary somatomotor cortices (S1fl) using two linear multi-electrode arrays (see Fig. 1a). The multi-electrode array has 23 contact points with a $100 \mu \mathrm{m}$ separation between each contact, which spanned the entire depth of the cortex (Einevoll et al. 2007). Laminar electrode arrays were located using a stereotaxic frame, and their depths were established by the laminar profile of evoked response (e.g., the earliest onset in evoked response along cortical depth). For validation, standard deviation in the mean depth of peak activation for evoked responses was calculated across rats. Standard deviation in the estimated depth between animals was 72 and $64 \mu \mathrm{m}$ for left and right $\mathrm{S} 1 \mathrm{fl}$, respectively, which was lower than standard deviation within individual rats (167 $\mu \mathrm{m}$ for both $\mathrm{S} 1 \mathrm{fl}$; see the online supplement). The extracellular recording signals were amplified and filtered between 0.1 and $500 \mathrm{~Hz}$ to record LFP. The LFP was recorded with a sampling rate of $2000 \mathrm{~Hz}$ under the following conditions: (1) for $10 \mathrm{~min}$ during rest and (2) for 4 min during forelimb stimulation $(\sim 1.2 \mathrm{~mA}, 3 \mathrm{~Hz}, 12$ pulses per train, duration of each pulse of $0.3 \mathrm{~ms}$, intertrain interval of 6-24 s).

\section{LFP data analysis}

All data analysis for the LFP signal was conducted using custom-written MATLAB code (The Mathworks; Natick, MA, USA). The LFP signal was preprocessed using a band-pass filter between 0.5 and $100 \mathrm{~Hz}$ to remove lowfrequency drifts and other noises. A band-stop filter between 59 and $61 \mathrm{~Hz}$ was applied to reject $60 \mathrm{~Hz}$ artifact. Burst suppression ratio was estimated similarly as in Vizuete et al. (2014) using above band-pass filter in order to examine burst suppression pattern in LFP activity.

The evoked responses were averaged at the onset of forelimb stimulation to exclude spontaneous background activity. Significant spontaneous activity was detected as positive and/or negative peak of larger than 2 standard deviations (SD) from the mean, and the boundary of each polarization was set as the points where all LFPs returned to a range within $1 \mathrm{SD}$ from the baseline. Each polarization was clearly distinguishable as shown in Fig. 1b. The peakto-peak amplitude of individual evoked response and spontaneous activity was estimated and averaged for each channel of electrodes (Fig. 1d). The type of each spontaneous activity was classified as biphasic if it contained both negative and positive peaks greater than $2 \mathrm{SD}$ from the mean. The spontaneous activity was classified as negative or positive if it had only negative or positive peaks, respectively. The cortical source density (CSD) was estimated by the second derivatives of the LFP signal along the cortical depth, which has been described in the earlier literature (Chapman et al. 1998). The CSD (in arbitrary unit) was numerically computed by taking a one-dimensional gradient twice using MATLAB.

The spectral power of the whole LFP signals at the cortical depths of 300, 700, and $1700 \mu \mathrm{m}$ was calculated using the Fast Fourier Transform algorithm in MATLAB for $4 \mathrm{~s}$ epochs during forelimb stimulation at $3 \mathrm{~Hz}$ (stimulation on period) and for $4 \mathrm{~s}$ epochs during inter-train intervals (stimulation-off period). The spectral power for each condition was averaged across epochs. The spectral power was also estimated for $4 \mathrm{~s}$ of averaged evoked response time series to remove out background spontaneous activity during stimulation on periods.

Independent component analysis (ICA) was applied to separate LFP activity into independent components, using the Fast ICA package 2.5 in MATLAB platform (Hyvarinen and Oja 1997). For evoked activity, ICA was conducted in the averaged evoked response (4 s epoch) as in the spectral power analysis. For spontaneous activity, ICA was conducted on the whole time course consisting of stimulation-off periods described in the above paragraph. The fast ICA algorithm utilized the principal component analysis (PCA) to reduce the data dimensionality. Then, ICA decomposed the LFP activity into four independent components (ICs), i.e., signal sources, which explained more than $98 \%$ of original signals (98.4 $\pm 0.6 \%$, mean $\pm \mathrm{SD})$. The ICs were independent to each other (Pearson's correlation $r<0.0001$ for all IC pairs), thus the total covariance was the sum of the covariance in each IC, from which the reconstructed correlation pattern was derived (see Online Resource).

The synchrony between LFP activity from the left and right S1fl was assessed with cross-correlation. The crosscorrelation between whole spontaneous LFP recordings from the bilateral S1fl was first estimated at each cortical depth (Fig. 6c). To estimate the interhemispheric delay, pairs of LFP polarizations in spontaneous activity of 

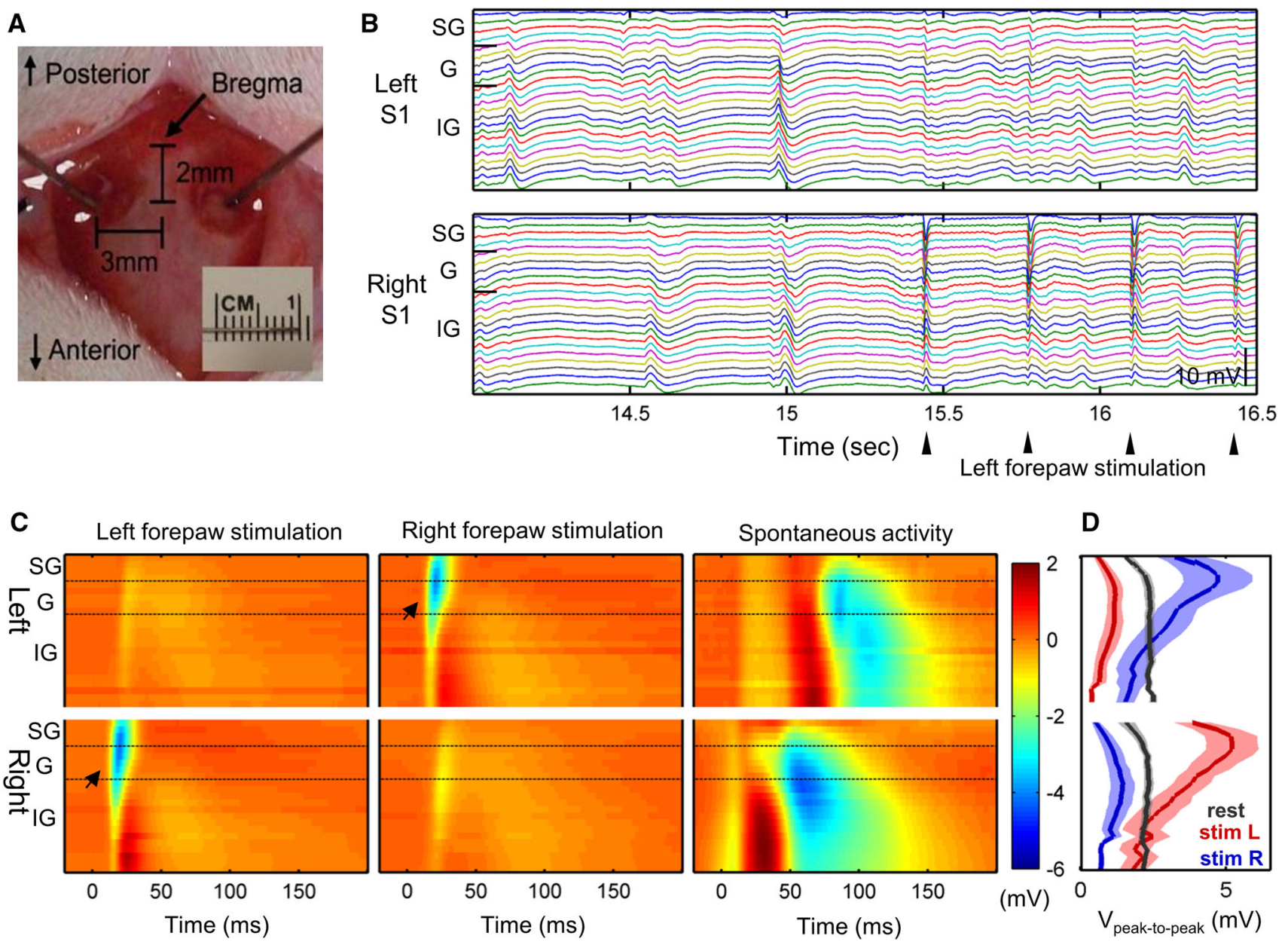

Fig. 1 Electrophysiological recordings from rat bilateral S1fl using laminar electrodes $(n=6)$. Two laminar electrodes were located on the forelimb regions of bilateral rat S1 cortices (a). Robust spontaneous activity was observed during rest and forelimb stimulation as shown in example of laminar electrode recordings (b). Averaged evoked response to left/right forelimb stimulation (left/ middle panels) and a typical example of spontaneous activity (right

panel) are shown in c. Arrow indicates the initiation of evoked response. d Peak-to-peak amplitude of spontaneous and evoked activities across the cortical depth (in $\mathrm{mV}$, mean $\pm \mathrm{SEM}$ ). $S G$ supragranular layer (layers 1-3), $G$ granular layer (layer 4), $I G$ infragranular layer (layers 5-6). Panels a, b, and $\mathbf{c}$ are typical examples from a representative rat

bilateral S1fl were identified, and the cross-correlation within a temporal range of -100 to 100 ms was estimated. For highly matched pairs of bilateral S1fl activity (Pearson's correlation $r>0.8$ ), the interhemispheric transfer delay was determined as the time delay yielding the maximal cross-correlation between bilateral LFP recordings.

\section{Magnetic resonance imaging experiment}

The resting state BOLD fMRI scans were performed for $10 \mathrm{~min}$, under the same anesthesia conditions as in the electrophysiology experiments (gradient echo planar imaging: $\mathrm{TR} / \mathrm{TE}=2000 / 25.93 \mathrm{~ms} ; \mathrm{FOV}=2.35 \times 2.35 \mathrm{~cm}$; matrix $96 \times 96$; nine contiguous $1 \mathrm{~mm}$ slices; $n=5$ rats). The MRI experiments were conducted using a $9.4 \mathrm{~T}$

horizontal bore (Magnex Scientific) scanner with a Bruker Avance console and custom-made surface-RF coil. Seven laminar region of interests (ROIs) along the cortical depth were drawn for each side of the somatosensory cortex (Fig. 6a), and the BOLD signal in each ROI was detrended and band-pass filtered $(0.01-0.1 \mathrm{~Hz})$ before the correlation analysis (Fig. 6b).

In addition, band-limited power (BLP) was calculated from the spontaneous LFP activity and convoluted with a canonical hemodynamic response function to be compared with rsfMRI signals. BLP is known to reflect slow modulation of spectral power of specific frequency band in the LFP recordings (Shmuel and Leopold 2008). The original broadband LFP recordings were band-pass-filtered using a fifth-order butterworth filter into the following frequency bands: delta $(0.5-4 \mathrm{~Hz})$, theta $(4-8 \mathrm{~Hz})$, alpha $(8-13 \mathrm{~Hz})$, 
beta $(13-30 \mathrm{~Hz})$, and gamma $(30-100 \mathrm{~Hz})$ band. Bandpass filtered signals were full-wave rectified and smoothed with a Gaussian window (FWHM: $250 \mathrm{~ms}$ ) to produce BLP time series (Nir et al. 2007). BLP time series was then convoluted with a canonical hemodynamic response function (Buxton et al. 2004) using a FWHM value of $1.92 \mathrm{~s}$ (Silva et al. 2007) and resampled into non-overlapping $2 \mathrm{~s}$ periods to match the sampling rate of the fMRI data. Then, we applied the band-pass filter of $0.01-0.1 \mathrm{~Hz}$ as same as in rsfMRI analysis. The zero-lag cross-correlation was calculated with the resulted BLP time series (i.e., simulated fMRI signals) as shown in Fig. 6e.

\section{Results}

\section{Neural basis of spontaneous neural activity}

As shown in Fig. 1a, using a pair of laminar electrode arrays which encompassed the whole cortical depth, the LFP activity was recorded in the forelimb regions of the bilateral primary somatosensory cortex (S1fl) of $\alpha$-chloralose anesthetized rats (Einevoll et al. 2007). We observed robust spontaneous activity during the resting state as well as evoked responses elicited by the electrical forelimb stimulation (Fig. 1b). Spontaneous activity can be characterized as a series of significant polarizations which occurred at the rate of $2.36 \pm 0.54 / \mathrm{s}$ (mean $\pm \mathrm{SD}$ ) without any prolonged period of burst suppression. The burst suppression ratio in spontaneous activity was negligibly small, i.e., $0.2 \pm 0.3 \%$ (mean $\pm \mathrm{SD})$.

The typical spatiotemporal patterns of the stimulationevoked response and spontaneous activity are shown in Fig. 1c. The forelimb stimulation induced brief, highly unilateralized evoked responses within a specifically confined spatiotemporal range. These stimulation-evoked responses originated at the granular layer 10-15 ms after the start of the forelimb stimulation, and rapidly propagated into the upper layers. This activation pattern is in accordance with the well-known thalamocortical afferent pathway which initially innervated to layer 4 (granular layer) and relayed to layers 2 and 3 (supragranular layer) (Einevoll et al. 2007). The evoked activity reached its peak at $25 \mathrm{~ms}$ after the forelimb stimulation (initial rise time of $\sim 10 \mathrm{~ms}$ ) and lasted $\sim 25 \mathrm{~ms}$ in the $\mathrm{S} 1 \mathrm{fl}$ contralateral to forepaw stimulation. The weaker ipsi-lateral responses were observed with around $8 \mathrm{~ms}$ interhemispheric delay after the contralateral activation. The peak-to-peak amplitude of the ipsi-lateral response was around $12-48 \%$ of the contralateral response $(24.6 \pm 15.1 \%$, mean $\pm \mathrm{SD})$. Both the ipsi- and contralateral evoked responses were followed by weak traces of synchronous polarization in the bilateral S1fl, which were nearly equal in amplitude and duration and shared the same termination (see Fig. 1c, left and middle panels), spreading into the infragranular layers of bilateral S1fl.

Compared with the consistency of stimulation-evoked responses, the spontaneous activity showed highly variable spatiotemporal profiles, which occurred across a broad cortical depth and often encompassed the deepest layer of neocortex. In general, the propagation rate and the duration of spontaneous activities were much slower and longer than the evoked responses. The spontaneous polarization returned to the baseline after $\sim 100 \mathrm{~ms}$ (taking up to $250 \mathrm{~ms}$ in some instances).

To characterize the laminar distribution of both the evoked and spontaneous neural activities, the peak-to-peak amplitudes along the cortical depth were evaluated (Fig. 1d). The evoked response was spatially confined to the upper layers of the contra-stimulus S1fl (mainly the granular and supragranular layers) where the thalamic afferent input preferentially projects. The maximal, contrastimulus, peak-to-peak response occurred at a depth of approximately $500 \mu \mathrm{m}$ from the cortical surface. The maximal, ipsi-stimulus response was found in the slightly deeper layers (i.e., at the border between layers 4 and 5). In contrast, the spontaneous activity exhibited a relatively stronger polarization in the infragranular layers (layer 5 and 6), and the maximal amplitude occurred at $\sim 1500 \mu \mathrm{m}$ or more from the cortical surface. The amplitude of the spontaneous activity was variable, and the mean amplitude was $\sim 60 \%$ of the stimulation-evoked response.

Spectral power analysis revealed that spontaneous activity occurring during the electrical forelimb stimulation had significantly decreased amplitude but unaffected frequency. As shown in Fig. 2a, the spectral power of spontaneous activity was concentrated at low frequencies (mostly between 1.5 and $2 \mathrm{~Hz}$ ) and decreased with $1 / \mathrm{f}$ distribution with frequencies higher than $2 \mathrm{~Hz}$ (see the online supplement), which resulted in low gamma power (30-100 Hz: $0.48 \%$ of the total power).

The power peaks of the evoked responses appeared as harmonics of $3 \mathrm{~Hz}$ (the frequency of the forelimb stimulation pulses), and thus, they were easily distinguishable from the spectral components of spontaneous activity. The low-frequency spectral power related to the spontaneous activity decreased during the forelimb stimulation, particularly in the contra-stimulus hemisphere (see Fig. 2). The reduction was prominent in the granular layers of the contra-stimulus S1fl (decreased to $\sim 38 \%$ of that measured without stimulation) where evoked response was strongest. Similar concomitant decreases of the spontaneous polarization reached deep into the infragranular layer (decreased to $\sim 74 \%$ ). The low-frequency spectral power in the ipsi-stimulus S1fl was relatively less affected by the forelimb stimulation with the peak reduction to the $74 \%$ compared to the resting condition. With additional signal 
Fig. 2 Spectral power distribution of spontaneous LFP activity (in $\mathrm{mV}^{2}$ ) and its modulation by sensory stimulation $(n=6)$. a Spectral power distribution of spontaneous LFP activity at the supragranular, granular, and infragranular layers $(300,700$, and $1700 \mu \mathrm{m}$ depth, respectively). A majority of spontaneous LFP power during stimulation-off blocks (blue, 'stim off') is concentrated in low frequency range of $0-5 \mathrm{~Hz}$. The power of the average evoked LFP responses (red, 'evoked') is exhibited as peaks of $3 \mathrm{~Hz}$ harmonics. The power of LFP activity during forelimb stimulation blocks (green, 'stim on') consists of both $3 \mathrm{~Hz}$ harmonics peaks and a lowfrequency component with decreased magnitude. b Modulation of low-frequency $(0-5 \mathrm{~Hz})$ power by sensory stimulation was profound in and around the granular layer of contra-stimulus hemisphere (mean \pm SEM). $S G$ supragranular layer (layers 1-3), $G$ granular layer (layer 4), $I G$ infragranular layer (layers 5-6)
A

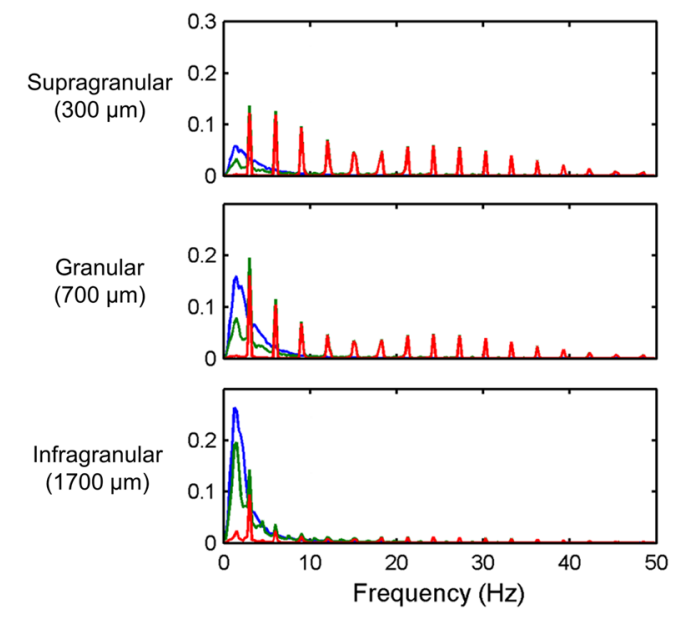

B

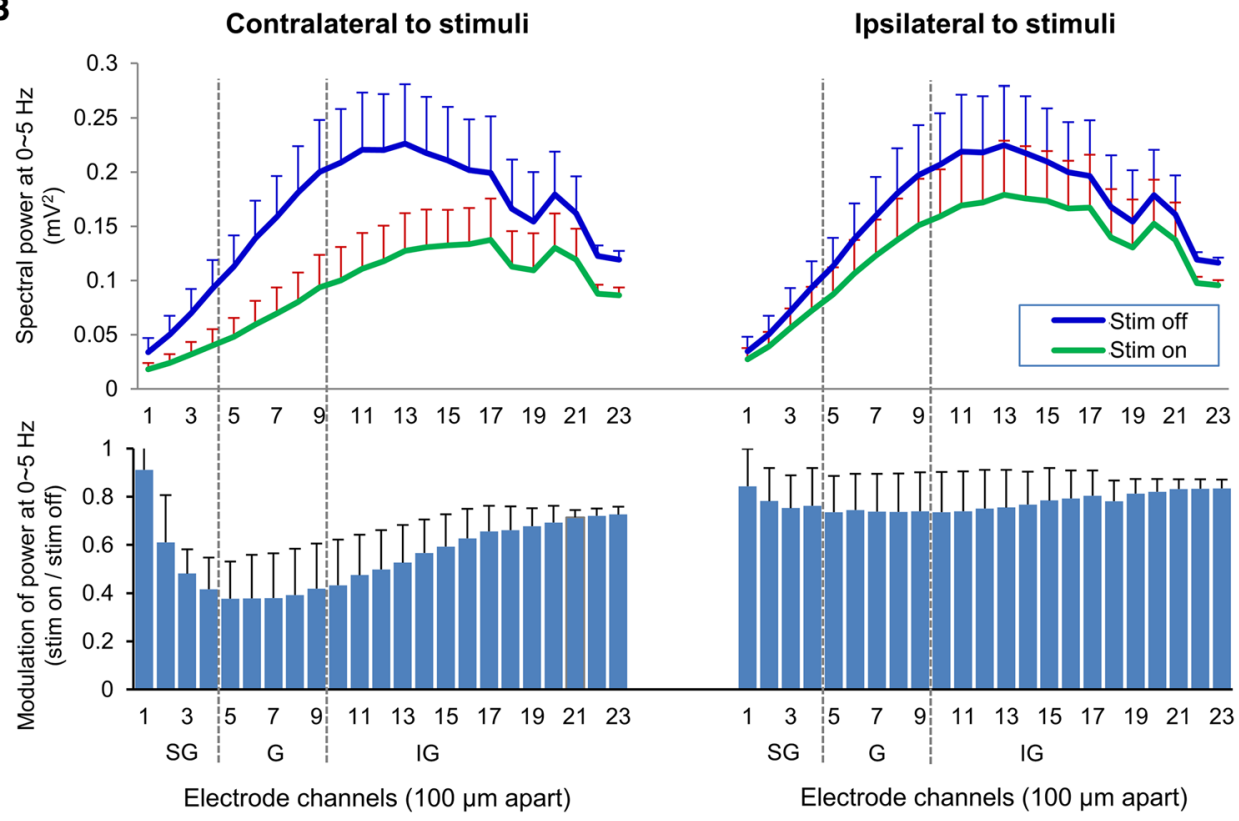

Ipsilateral to stimuli
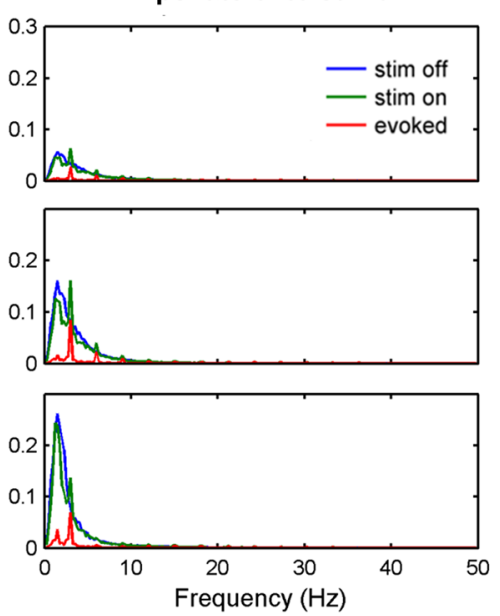

Ipsilateral to stimuli 


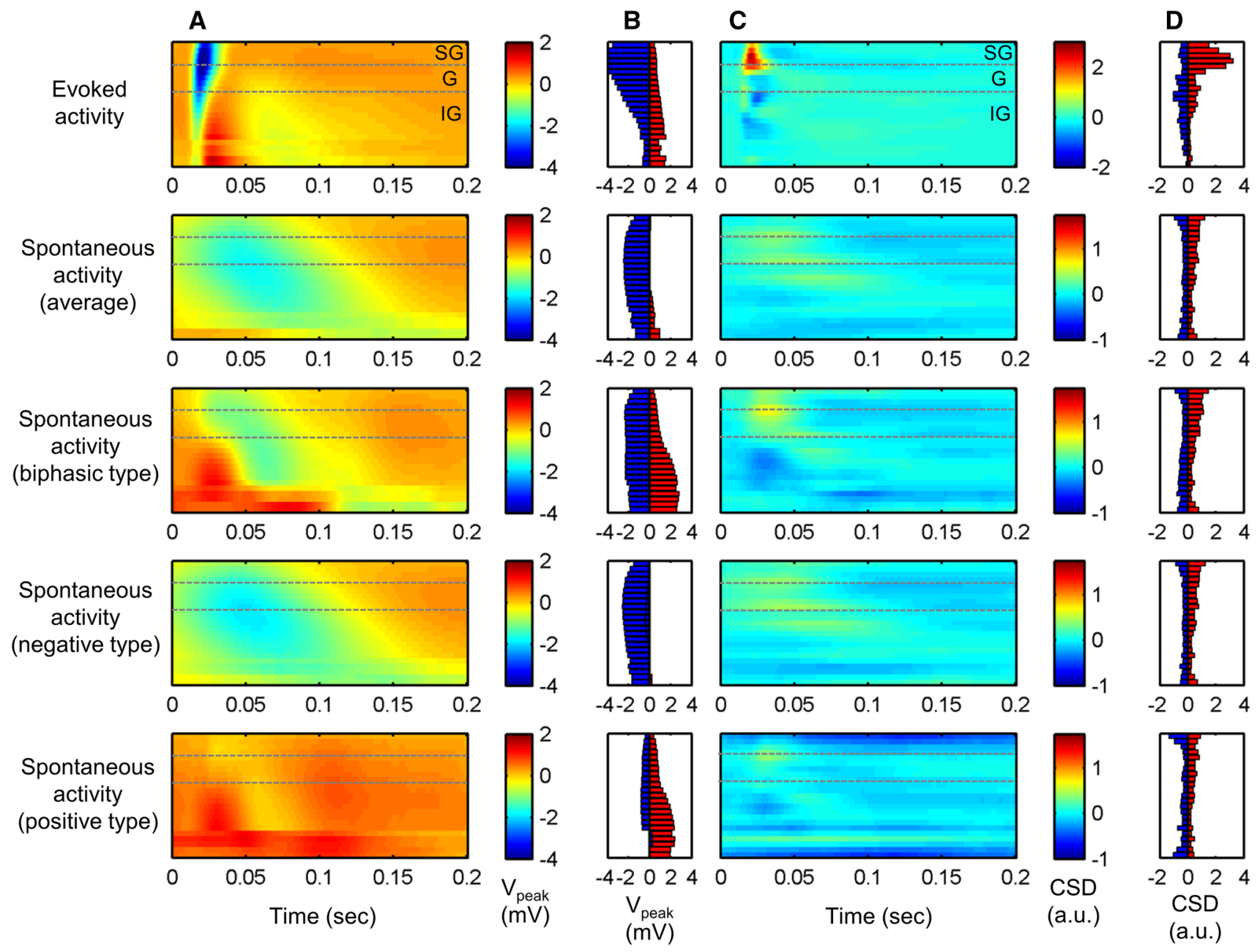

Fig. 3 LFP polarization and current source density $(C S D)$ of evoked activity and each type of spontaneous activity $(n=6)$. The averaged LFP activity profile (in $\mathrm{mV}$ ) revealed slower dynamics in spontaneous activities (a). Spontaneous activity, particularly biphasic type, tends to be stronger in granular and infragranular layers (b). Positive (red) and negative (blue) peak voltage of LFP (in $\mathrm{mV}$ ). Spontaneous

activity had gradual current sink and sources in relatively lower locations than evoked activity as shown in averaged cortical source density profile (c) and peak magnitude of current sink and source (d). Current sink is depicted as positive value (red) and source as negative (blue) in arbitrary unit

current sinks and sources in the spontaneous activity were also dynamically slower and relatively weaker than those associated with the evoked activity, implying the involvement of sparse synaptic currents.

We applied independent component analysis (ICA) in order to decompose both evoked and spontaneous activity into tentative signal sources which are independent to each other. The ICA assumed that the observed multi-channel LFP signal was a weighted sum of several signal sources, which were differently weighted along the cortical depth. The original LFP signals during resting state were separated into four main independent components (ICs), which accounted for $98.2 \pm 0.8 \%$ (mean $\pm \mathrm{SD}$ ) of the original signal (see Fig. 4h). The averaged evoked activity during the forelimb stimulation was also successfully divided into four ICs (Fig. 4d), which explained $98.6 \pm 0.4 \%$ of the

signal. These independent components were ordered by the amount of contribution in the original LFP signal (e.g., IC 1 explained the greatest variance of the LFP signal) as shown in Fig. 4.

Among the four main components in the evoked response (Fig. 4a-d), the IC 1 indicated the dynamically slow component, which was not directly linked to the apparently evoked response pattern. This result likely represents residual spontaneous activity in background. The IC 2 and IC 3 were more directly tied to the evoked responses, in which the IC 3 exhibited the initial, sharp rise in evoked responses, concentrated in the granular layer of the contra-stimulus S1fl region. Meanwhile, the IC 2 reflected subsequent activations in the supragranular layer in both the contra-stimulus and ipsi-stimulus S1fl regions. The profiles of ICs 2 and 3 activations were in accord with 
Fig. 4 Decomposition of evoked and spontaneous activities using independent component analysis (ICA). Both evoked and spontaneous activity were decomposed into four independent components $\left(I C_{k}\right)$, respectively $(\mathbf{a}, \mathbf{e}) . \mathrm{IC}_{\mathrm{k}}$ are depicted in order of contribution from largest to smallest. Panels $\mathbf{b}$ and $\mathbf{f}$ exhibit basis vector $a_{\mathrm{k}}$ for kth independent component, representing the weight of each independent source along the bilateral cortical layers. LFP signal reconstructed by summing $\mathrm{IC}_{1}$ to $\mathrm{IC}_{\mathrm{k}}$. Four ICs have successfully reconstructed the original signals $(\mathbf{c}, \mathbf{g})$, and residual errors in the reconstructed signal (red total signal variance, white unexplained variance) are shown in panels $\mathbf{d}$ and $\mathbf{h}$. Data from a representative animal
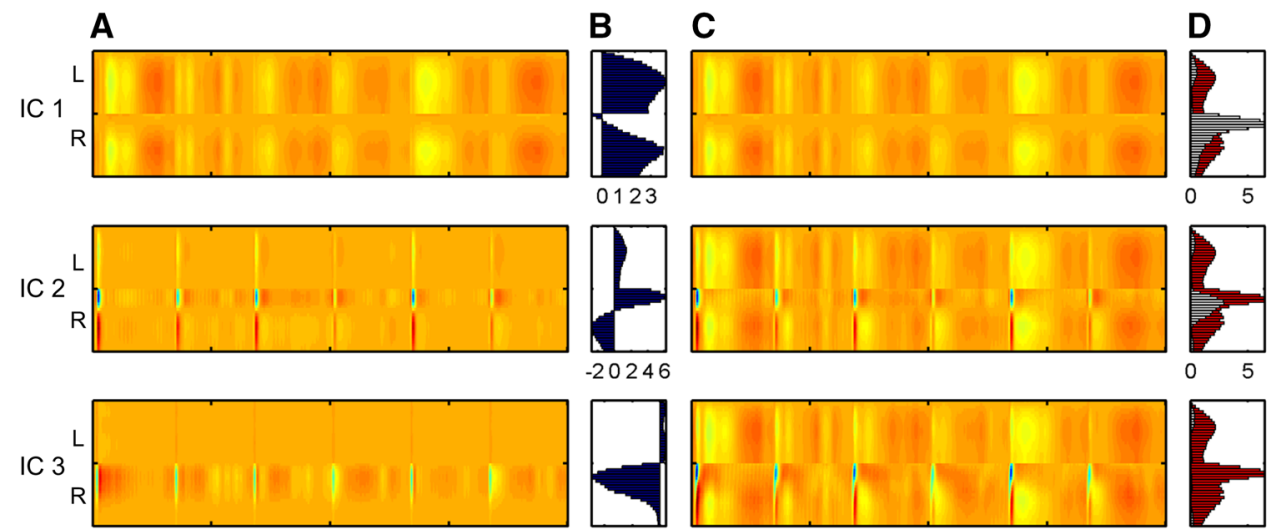

$-20246$
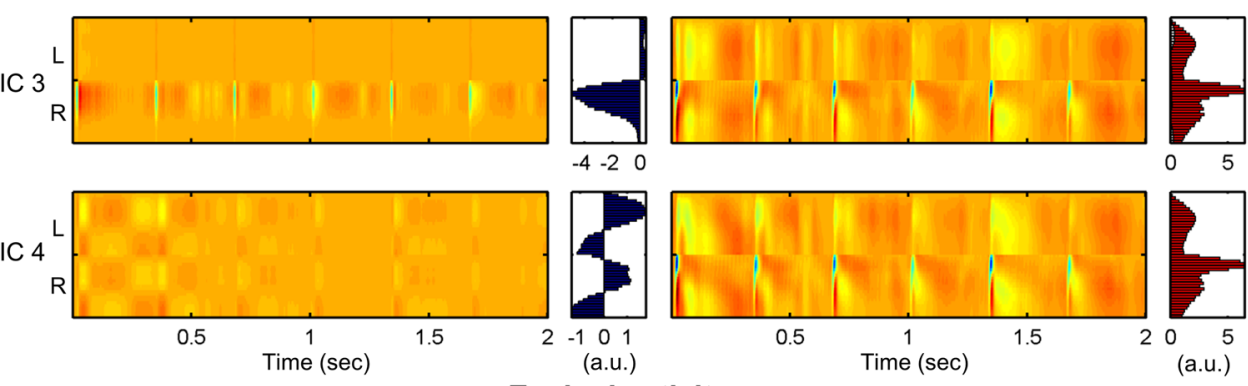

Evoked activity
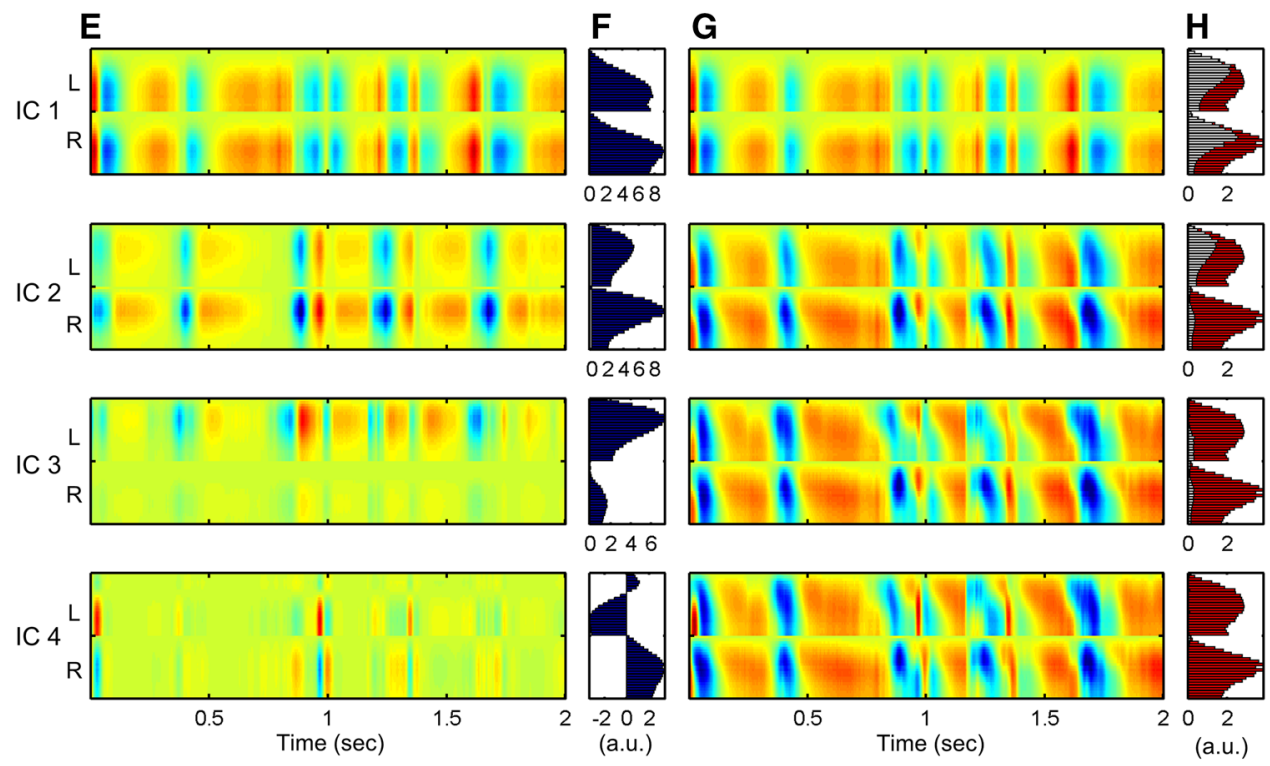

Spontaneous activity the known "canonical microcircuit" for thalamic afferent input (Lubke and Feldmeyer 2007) and successfully dissociated the granular and supragranular populations involved in the evoked response. Finally, the IC 4 exhibited as the bilaterally synchronous signals differently polarized between the upper (supragranular and granular) and infragranular layers, which might contribute to weak trailing polarizations spreading across depth in the bilateral S1f1. The spontaneous activity was also divided into four main independent components as shown in Fig. $4 \mathrm{e}-\mathrm{h}$. The primary component covering the largest fraction (i.e., IC 1) was bilaterally synchronized activities, found mostly in the infragranular layer. This IC 1 in spontaneous activity was very similar to the IC 1 in the evoked activity in terms of spatiotemporal profile, thus might reflect the same bilateral signal source. In contrast, ICs 2 and 3 were lateralized and concentrated in upper layers. The IC 4 of the spontaneous activity was concentrated in lower layers and negatively correlated between bilateral $\mathrm{S} 1 \mathrm{fl}$ regions.

\section{Interhemispheric synchronization}

In contrast to the highly lateralized evoked response, spontaneous activity was largely synchronous between the left and right S1fl as shown in Fig. 1. A significant fraction of the spontaneous activity bilaterally occurred in a mirrored pattern with comparable amplitudes but variable interhemispheric time delays, up to $\sim 50 \mathrm{~ms}$ (for example, 
see Fig. 1c). The interhemispheric correlation of spontaneous activity was particularly strong in deeper layers and was around the zero-lag (Fig. 5a). Additionally, for quantitative estimation, each pair of spontaneous activity in the bilateral S1fl was identified between which the cross-correlation values were calculated. As reflected in the strong correlation values, a majority of interhemispheric time delays were also found around the zero delay (Fig. 5b). The interhemispheric time delays for the highly matched pairs of activity (Pearson's correlation $r>0.8$ ) are shown in Fig. 5c, d. Approximately, $40 \%$ of the synchronized pairs were found at the zero-lag while the interhemispheric delay of the remaining cases was distributed in the range of -30 to $+40 \mathrm{~ms}$. In about a half of the animals, the distribution of interhemispheric delays were slightly biased in the direction from the right hemisphere to the left.
The evoked activity exhibited relatively low but nonzero interhemispheric correlation with a strictly fixed interhemispheric time delay. As shown in Fig. 5e, the interhemispheric correlation in evoked activity was highest with the interhemispheric delay of $\sim 8 \mathrm{~ms}$ (particularly at the granular and the supragranular layers). Meanwhile, the infragranular layer was also found highly correlated around the zero-lag, which was most likely influenced by the spontaneous background activity. The delay between each individual evoked response pair was also calculated independently in which most of the interhemispheric time delays were also estimated to be $\sim 8 \mathrm{~ms}$ (Fig. 5f).

Upon zero-lag correlations of the spontaneous activities measured at different cortical depths within and between hemispheres (Kim et al. 2008), we found layer-specific correlations between the bilateral cortices. That is, LFP
Fig. 5 Interhemispheric delay between bilateral S1fl for both spontaneous and evoked activities $(n=6)$. $S G$ supragranular layer (layers 1-3), $G$ granular layer (layer 4), $I G$ infragranular layer (layers 5-6). Spontaneous activity in the bilateral S1fl was mostly synchronized with a delay below $\sim 5 \mathrm{~ms}$, particularly in lower layers, as shown in averaged cross-correlation between spontaneous LFP recordings from bilateral electrodes (a). Highly correlated pairs of activities tended to have relatively short interhemispheric delays (b). Distribution of the interhemispheric time delay in spontaneous activity (mean $\pm \mathrm{SD}$ ) in group mean and each animals are shown in panels $\mathbf{c}$ and $\mathbf{d}$. Unlike spontaneous activity, evoked activity in the bilateral S1fl was synchronized with a delay of $\sim 8 \mathrm{~ms}$ and was particularly prominent in the middle layers (e). A large fraction of evoked activity tended to show a fixed delay of $\sim 8 \mathrm{~ms}$ (f)
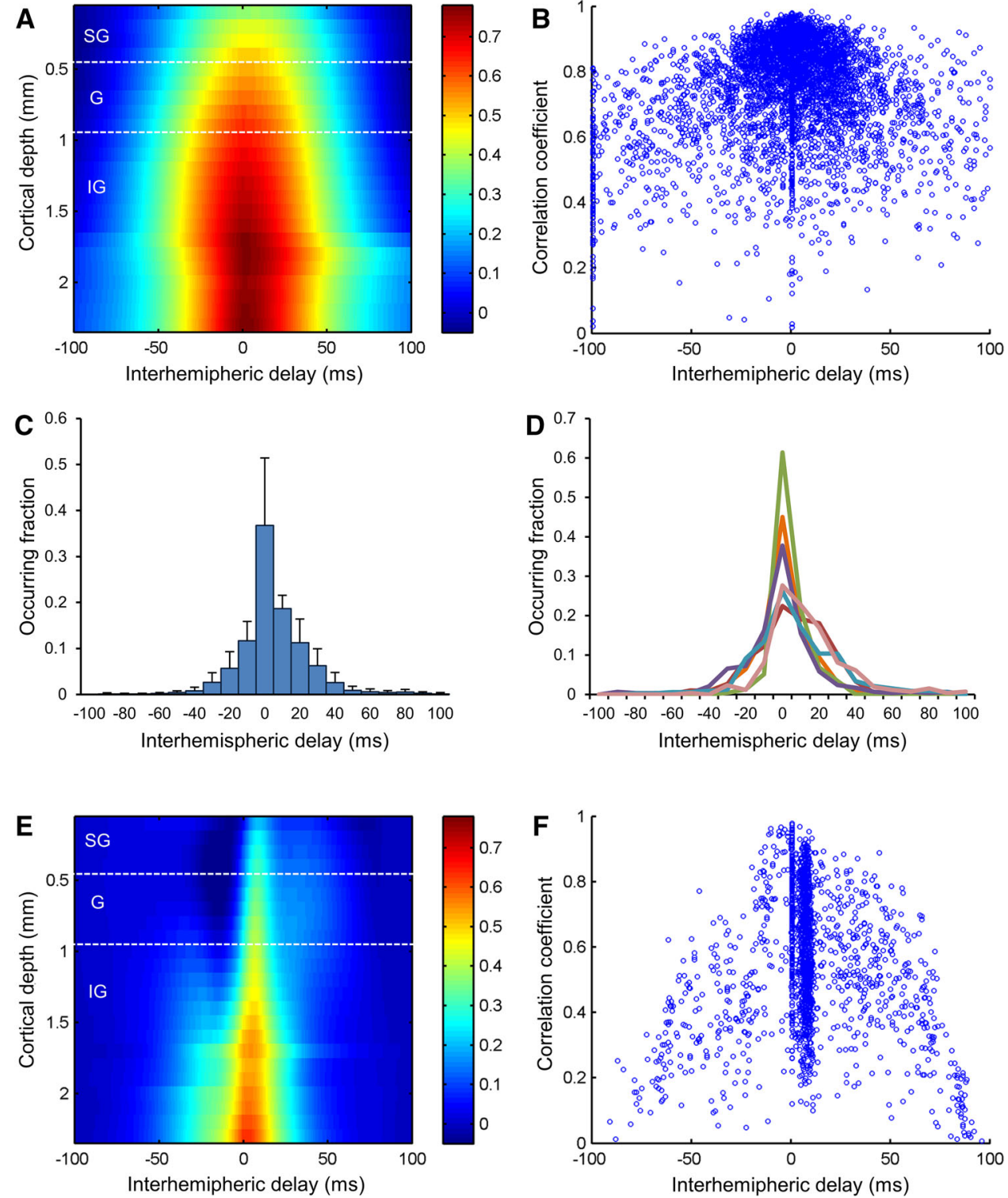
recordings from the same depths in the bilateral S1fl were more strongly correlated than those acquired at different depth levels, which resulted in a diagonal distribution of prominent cross-correlation (Fig. 6c). In addition, the correlation coefficients were relatively higher between the lower layers compared with the upper layers. The observed layer-specific pattern was well supported by the covariance structure of the independent components (see Online Resource) acquired from the spontaneous LFP signals. IC 1 largely contributed interhemispheric correlation particularly in lower layers, and IC 2/3 exhibited weak covariation in upper layers of bilateral S1fl.

Figure $6 \mathrm{~b}$ shows the representative BOLD rsfMRI time series, from which the functional connectivity pattern between the bilateral S1 regions (Fig. 6d) was created using the same zero-lag cross-correlation as in Fig. 6c. The spontaneous BOLD rsfMRI signals also exhibited the layer-specific correlation pattern, similar to the results from the laminar LFP recordings. However, unlike electrophysiology, the rsfMRI signals exhibited high interhemispheric correlation was also observed between upper layers as well. Negative correlation between distant cortical depths (i.e., upper vs. lower layers) was also observed in rsfMRI. Still, these two 2-D correlation maps from LFP activity and rsfMRI signals (Fig. 6c, d) were highly similar, showing Pearson's correlation $r=0.47$ $(p<0.001$, see the online supplement) between correlation maps from these two modalities.

The BLP time series for five spectral bands were calculated from LFP recordings and convoluted with a hemodynamic response function in order to be compared with the rsfMRI results. The layer-specific interhemispheric correlation patterns observed in theta, alpha, and beta BLP paralleled that found in the rsfMRI experiment (Pearson's correlation between correlation maps $r=0.44$, 0.69 and 0.41 , respectively; all $p<0.01$. see the online supplement), but gamma and delta BLP did not show such similarity as shown in Fig. 6e.

\section{Discussion}

\section{Dissociation of spontaneous activity from evoked responses}

The robust spontaneous neural activity in the rat S1fl and its synchronization between bilateral hemispheres represent
Fig. 6 Synchronization across cortical depth within and between the left/right $\mathrm{S} 1$ cortices $(n=6$ for LFP recordings, $n=5$ for resting state fMRI). Seven laminar ROIs were defined for each side of the somatosensory cortex (a) and resting state BOLD fMRI signals from the laminar ROIs were extracted (b). Layerspecific pattern was observed in zero-lag cross-correlation between bilateral S1 activity measured in both LFP

recordings (c) and resting state BOLD fMRI (d). Note that the correlation coefficient values were normalized using Fisher's $r$-to- $z$ transformation. The bandlimited power (BLP) time series were calculated from the LFP recordings and convoluted with hemodynamic response function to be compared with the resting state fMRI signals. Alpha and beta BLP convoluted with a hemodynamic response function replicated the laminar-specific correlation pattern observed in resting state fMRI (e). Panels a, $\mathbf{b}$, and $\mathbf{e}$ are examples from a representative rat
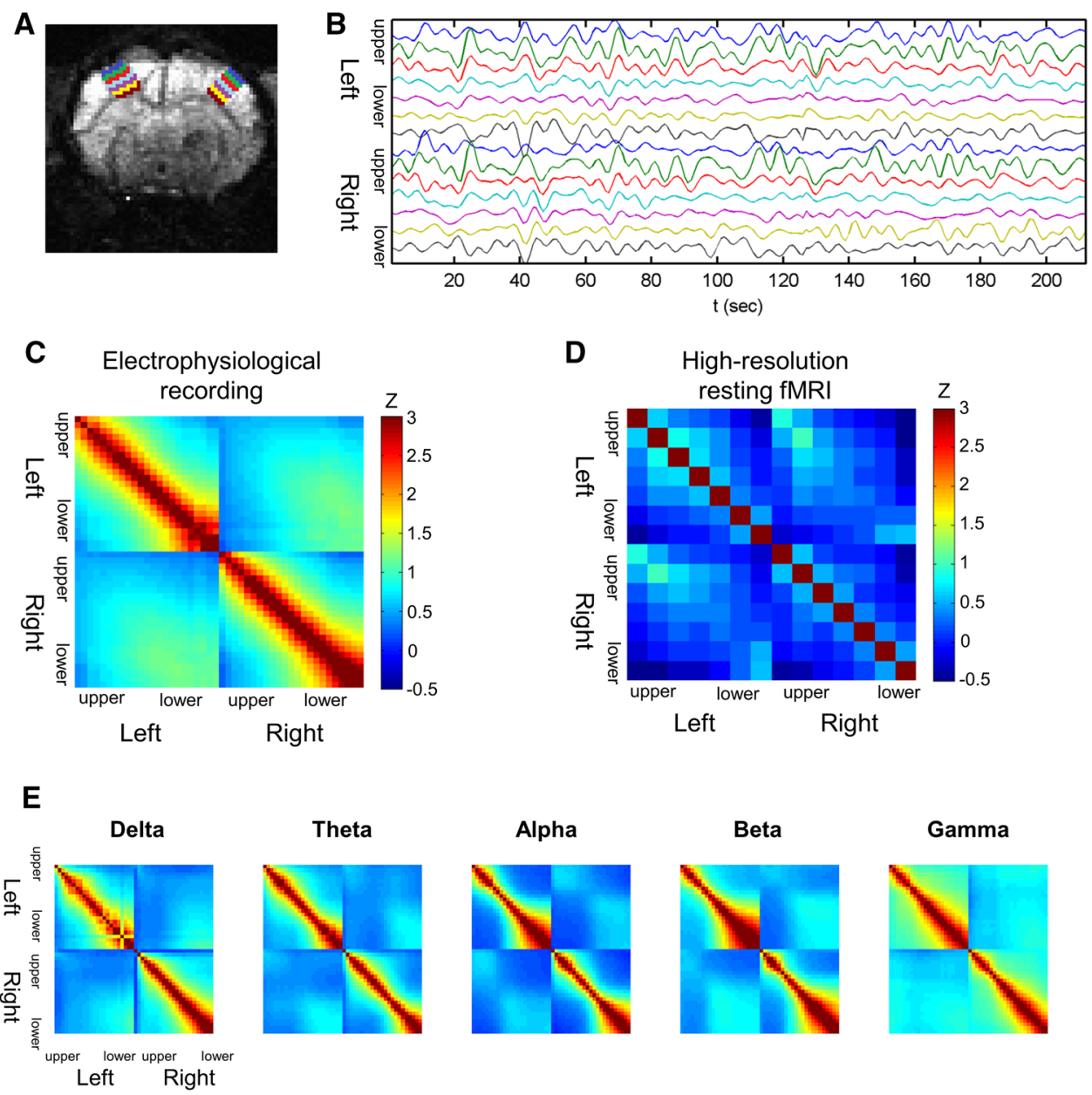

Theta
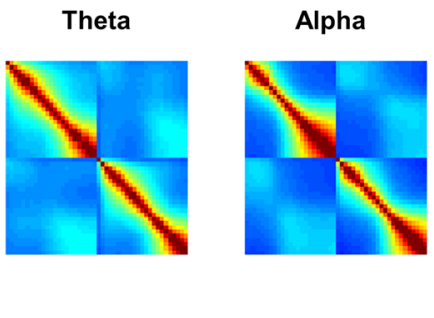

Gamma
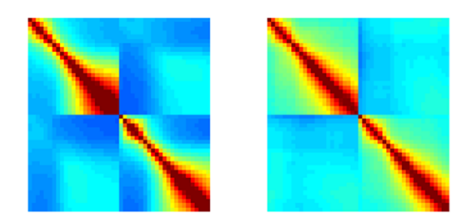
the building blocks of the interhemispheric cortical connectivity. In particular, the remarkable similarity in the layer-specific correlation patterns between electrophysiology and rsfMRI likely provides the neural basis of functional connectivity observed in the previous rsfMRI studies (Biswal et al. 1995; Fox et al. 2005; Johnston et al. 2008; Lowe et al. 1998; Quigley et al. 2003). Moreover, in good agreement with the previous functional magnetic resonance imaging (fMRI) observation by Fox et al. (2005), the evoked neural responses to sensory stimulations also appeared superimposed on a background of spontaneous activity. Despite the modulation of the signal amplitude at the supragranular layer, the frequency of spontaneous activity and its spatiotemporal pattern was little affected by the presence of evoked responses. This particular dissociation implies that spontaneous activity was derived from the specific neuronal populations that are functionally independent from those involved in the evoked activity.

The spectral power of spontaneous activity was concentrated in delta and theta band ranges $(0.5-4$ and $4-8 \mathrm{~Hz}$, respectively) and peaked at $\sim 2 \mathrm{~Hz}$, approximate occurrence rate of distinguishable polarizations in the spontaneous activity. Delta band activity was found to be dominant in spontaneous LFP activity in both primates and rodents (Leopold et al. 2003; Lu et al. 2007). However, unlike the previous reports in human and animal studies (Scholvinck et al. 2010; Shmuel and Leopold 2008; Nir et al. 2008; Magri et al. 2012; Thompson et al. 2013), we could not find relevant slow modulation $(<0.1 \mathrm{~Hz})$ of gamma BLP, most likely due to relatively small contribution of gamma band activity in the rat brain (i.e., $0.48 \%$ in the present study vs. $3.49 \%$ in the human, approximated from the report by Nir et al. (2007)). Gamma BLP has been most frequently indicated as neural basis of rsfMRI signals (Shmuel and Leopold 2008; Scholvinck et al. 2010; Thompson et al. 2013; Magri et al. 2012), but delta and theta bands have been also implicated in spontaneous brain activity and rsfMRI (Scholvinck et al. 2010; Pan et al. 2010, 2011; Leopold et al. 2003). Delta and broadband $(1-100 \mathrm{~Hz})$ power modulation was found to be correlated with rsfMRI signals of rats under isoflurane anesthesia (Pan et al. 2010, 2011). Delta and theta BLP were also most strongly coherent with BOLD rsfMRI signals of rats anesthetized with dexmedetomidine (Pan et al. 2013). More relevantly, delta oscillations were dominant in the rat brains during $\alpha$-chloralose anesthesia regardless of dose and responsible for functional connectivity between bilateral rat S1fl (Lu et al. 2007).

As for the possible effects from anesthetics, $\alpha$-chloralose is one of the most widely used anesthetics in fMRI experiment in rodents and has been found to preserve the specific functional BOLD response and functional connectivity patterns when compared to other anesthetics such as isoflurane (Williams et al. 2010; Peeters et al. 2001; Majeed et al. 2009). We also tried to use the minimal dose of $\alpha$-chloralose in the present study (a loading dose of $50 \mathrm{mg} / \mathrm{kg}$ and continuous intravenous infusion at $40 \mathrm{mg} /$ $\mathrm{kg} / \mathrm{h}$ compared to 80 and $30 \mathrm{mg} / \mathrm{kg}$ in Lu et al. 2007). LFP recordings in the present study exhibited the negligible amount of burst suppression, periods of strong burst activity alternating with silent periods, in contrast to the deep anesthesia induced by isoflurane (Liu et al. 2011). Finally, contribution of delta band in rsfMRI and functional connectivity was suggested in above animal studies using different types of anesthetics (Lu et al. 2007; Scholvinck et al. 2010; Pan et al. 2011; Magnuson et al. 2014).

Previous studies also revealed that slow spontaneous deflections in LFP signal align with cortical 'up' and 'down' states (Csercsa et al. 2010; Haslinger et al. 2006). In accordance with the high spontaneous LFP activity in infragranular layers, such slow oscillations have also been reported to be prominent in lower cortical layers with their phases linked with excitability of the cortical neurons (Haslinger et al. 2006). These types of slow oscillations have been mainly observed during slow wave sleep and under anesthesia. Although such coordinated activity of neurons communicating via synapses within recurrently connected networks is thought to underlie the spontaneous neocortical activity, the exact neural mechanisms of 'up' and 'down' state oscillations are not clear and are still under investigation. Apart from such hypotheses, the current study clearly demonstrated that the spontaneous cortical activity itself is highly synchronized between bilateral hemispheres.

\section{Laminar population underlying evoked and spontaneous activities}

Spontaneous activity exhibited slower dynamic features compared to the evoked activity. The electrical forelimb stimulation induced a neural response that rapidly reached its peak with a rise time of $\sim 10 \mathrm{~ms}$, whereas the rise time of a single spontaneous activity was much longer $(>25 \mathrm{~ms})$ with a duration of 100-250 ms. In theory, the long-lasting dynamic parameters are possibly due to the hyperexcitability of infragranular neurons (i.e., L5) which are intrinsically more depolarized than supragranular neurons (i.e., L2/3 neurons), which are $\sim 10 \mathrm{mV}$ closer to the action potential threshold (Lefort et al. 2009). In addition, previous studies also revealed the tight synaptic coupling among L5 neurons in a highly recurrent excitatory microcircuit (Bannister 2005; Crochet and Petersen 2009). Such hyperexcitability combined with the extended synaptic activity of infragranular neurons might account for slow waves of excitation, resulting in the slow dynamic features of spontaneous activity observed in this study. 
In CSD analysis of evoked activity, we revealed the initial current sink in L4 followed by a strong current sink at L2/3, which is in accord with well-known thalamocortical afferent pathway, i.e., initial afferent input in L4 followed by strong activation of L2/3. On the other hand, as previous studies have suggested synchronized synaptic inputs to the infragranular layer (Crochet and Petersen 2009), the initial cellular excitation in L5 and L6 accounted for the majority of the spontaneous LFP activity and the associated connectivity. In general, current sinks and sources in the spontaneous activity were located only slightly deeper than and largely overlapped with those found in evoked activity. However, near complete exclusion of the upper supragranular layer activity $(\sim 200 \mu \mathrm{m})$ along with manifold spatiotemporal characteristics demonstrated the uniqueness of dendritic and neuronal populations associated with spontaneous activity, set apart from those involved in evoked activity. Although incomplete to explain all the associated phenotypes (e.g., absence of superficial layer activity), such apparent spontaneous CSD activity in the layers $2 / 3$ and 4 was likely due to the pyramidal neurons in layer 5 and 6 , which often have their dendritic tree extended up to the supragranular layer (Lubke and Feldmeyer 2007; Douglas and Martin 2004). In this regard, the spontaneous synaptic events in the upper cortical layers were most likely derived from the aforementioned afferent inputs to the infragranular neurons.

The ICA divided both evoked and spontaneous activities into four tentative signal sources, which constituted more than $98 \%$ of the original signals. In evoked activity, ICs 2 and 3 were prevalent in the contra-stimulus layers $2 / 3$ and 4, which specifically divided the stimulus-induced responses activated via a well-known thalamocortical pathway. Contrary to the highly lateralized ICs 2 and 3, ICs 1 and 4 were nearly equivalent between bilateral hemispheres. Based on the spatial weight profile, IC 1 of the evoked activity likely accounted for activities in layer $5 / 6$ neurons while IC 4 accounted for weak residual signal propagation across cortical depth into the infragranular layers. Similar to the IC 1 with an evoked response, the primary component (IC 1) in spontaneous activity isolated bilaterally synchronized signal source concentrated in the infragranular layers. Such bilateral activity in the infragranular layer might be largely responsible for the observed interhemispheric synchronization. There were also considerable unilateral spontaneous activities in the upper layers (IC 2, 3), which likely contributed to the relatively weak correlation between the upper layers of bilateral S1fl. The anti-correlated IC 4 might be related to the variable interhemispheric delays. When considering all the evidence, the spontaneous activity was primarily driven by the bilaterally synchronized signal sources concentrated in the deeper cortical layers and was modulated by the unilateral activity in the upper layers and interhemispheric delay component. The tentative dissociation of the LFP activity between upper and lower layers was also previously demonstrated by Maier et al. (2010) using laminar coherence analysis.

\section{Interhemispheric communication and anatomical connections}

Markedly different interhemispheric neural characteristics were observed between the spontaneous and evoked signals, which indicates that independent neural mechanisms govern these two interhemispheric synchronization processes. Although the evoked response was highly lateralized, the weak ipsi-stimulus S1fl responses were consistently present. The interhemispheric delay in the ipsistimulus S1fl was stable at $\sim 8 \mathrm{~ms}$, which is comparable to the transcallosal conduction delay measured in the previous electrophysiological experiment (Seggie and Berry 1972). On the other hand, the spontaneous activity was highly correlated across the bilateral S1fl, with a comparable amplitude and spatiotemporal pattern. Unlike the evoked response, the time delay between the bilateral pairs was apparent and highly variable as shown in Fig. 5b, despite the bilateral similarity in the activity profile. Given the high bilateral similarity of spontaneous activity in a pairwise pattern, bilateral S1fl most likely shared the same underlying neuronal populations and afferent inputs unique for each subtype.

The corpus callosum is a key candidate for the anatomical site mediating the interhemispheric communication between cortical regions. The callosal fibers are known to innervate layers 2-6 of the neocortex (densely to layers 3 and 5 but sparsely to layer 4) (Wise 1975; Isseroff et al. 1984; Hayama and Ogawa 1997). The role of the corpus callosum has been demonstrated in previous functional connectivity studies which involved disruption of the corpus callosum connectivity in animals (Mohajerani et al. 2010; Magnuson et al. 2014) and humans (Johnston et al. 2008; Quigley et al. 2003). While the ipsi-lateral stimulusevoked activity was most likely mediated by the corpus callosum in the present study, the interhemispheric conduction delay in spontaneous activities was estimated to vary by up to $\sim 50 \mathrm{~ms}$, which makes the direct transmission via the corpus callosum unlikely. Moreover, a significant fraction of the spontaneous activity was synchronized at the zero time lag. Thus, the interhemispheric correlation in spontaneous activity might not simply arise from the unidirectional transcallosal transmission.

On the other hand, the cortical column is massively interconnected with subcortical structures, such as the thalamus, striatum, and brainstem. Among these connections, the thalamocortical loop has been considered as a 
putative source of oscillatory activity in the neocortex and possibly a potential mediator of the interhemispheric functional connectivity (Lumer et al. 1997). In agreement with the heightened spontaneous activity in deep cortical layers, the infragranular layer is known to receive thalamic afferent inputs. In particular, layer 6 is reciprocally connected with the thalamic nuclei (Lubke and Feldmeyer 2007). Although the common afferent pathway to bilateral $\mathrm{S} 1 \mathrm{fl}$ is a strong possible source of the manifest spontaneous synchronicity, highly variable spatiotemporal patterns (Fig. 3) and long interhemispheric delays weaken this hypothesis. Nonetheless, the current study revealed that spontaneous cortical activity has multiple neural processes and conduction pathways (Uddin et al. 2008), which recruit variable neural populations resulting in highly irregular activity patterns with variable delays between each bilateral pair. Magnuson et al. (2014) found significantly reduced but partially preserved interhemispheric functional connectivity in rats with full callosotomy, supporting that polysynaptic pathways mediate interhemispheric communication.

\section{Layer-specific interhemispheric synchronization in electrophysiology and rsfMRI}

A layer-specific pattern of zero-lag correlation has been first identified in spontaneous LFP activity. Although this finding may simply suggest a fine laminar arrangement of the interhemispheric connections, the laminar-specific correlation pattern was likely derived from the independence of activities between the lower (infragranular) and upper (granular/supragranular) layers. For example, in contrast to the highly synchronous bilateral activity in the infragranular layer, sporadic unilateral activity in the granular/supragranular layer appears to influence the correlation pattern. Such lateralized signals also contribute to the reduced synchronization with the upper layers. In agreement with this evidence, the spontaneous activity comprises independent components that are well divided between the lower (IC 1) and upper layers (ICs 2 and 3). As shown in the ICA correlation (see the online supplement), the covariance of IC 1 demonstrated the high synchronous activity in the infragranular layer, and ICs 2 and 3 showed weaker but similar patterns in the upper layers. In particular, the patterns from ICs 2 and 3 were linked with unilateral activity in the upper layers, which subsequently contributed to the weak interhemispheric correlation between bilateral upper cortical layers as well as the weak correlation between upper and infragranular layers. The different ICA correlations strongly suggest that independent inputs to upper and lower layers are responsible for the layer-specific pattern observed in the study.
We also found a similar layer-specific correlation pattern in our high-resolution rsfMRI signals. Although speculative in this early stage, it remains an interesting question how functional connectivity between distant brain regions arises with regard to the well-known laminar organization of the neocortex.

However, there were a few differences between the electrophysiological recordings and rsfMRI data. The rsfMRI data showed positive correlations between the upper layers and significant negative correlations between upper and lower layers. The difference in temporal scale between rsfMRI and the electrophysiological recording can primarily account for these difference as shown in our results of BLP convoluted with a hemodynamic response function, particularly for alpha and beta BLP. It clearly shows a role of hemodynamic response function in the characteristics of rsfMRI signals, although we failed to show layer-specific correlation in gamma band power modulation, which has been frequently reported in previous literatures (Nir et al. 2007; Shmuel and Leopold 2008; Scholvinck et al. 2010; Magri et al. 2012; Thompson et al. 2013; Pan et al. 2011). In addition, rsfMRI can be affected by the characteristics of the vascular structure and neurovascular coupling along in the cortical depth (Goense et al. 2012; Tian et al. 2010; Silva and Koretsky 2002; Harel et al. 2006). For example, evoked BOLD responses are reported to be the strongest at the cortical surface due to the presence of relatively large blood volume and venous drainage (Tian et al. 2010; Mandeville et al. 2001; Harel et al. 2006), and our rsfMRI signals in laminar ROIs were stronger in upper layers as well as shown in Fig. 6b. This difference in the magnitude of vascular responses might have exaggerated the interhemispheric correlation in the superficial layers as a result of increased signal-to-noise ratio. To further elucidate, the initial dip in the BOLD fMRI signal is found only in the superficial layer due to the delayed vasodilation of the capillary bed (Tian et al. 2010). This delayed vasodilation may also account for the negative correlations between the spontaneous BOLD signals in the upper and lower cortical layers. Additionally, other unknown physiological issues may come into play, including a possibility that the mechanism of neurovascular coupling involved in spontaneous activity is diverse and entirely different from that involved in evoked activity. Despite these uncertainties, the layer-specific interhemispheric correlations (with electrophysiology and rsfMRI) identified the spontaneous neural activity that underlies the observed cerebrohemodynamic activity. Further study using both modalities will be required to elucidate the relationship between the layer-specific pattern observed in laminar electrophysiological recordings and BOLD rsfMRI. 


\section{Conclusion}

In summary, the current study identified significant interhemispheric correlations in both electrophysiological and hemodynamic rsfMRI signals. The ICA suggested that there are independent neural inputs to infragranular and granular/supragranular layers with a difference in laterality between the input groups. These distinctive inputs likely determine the spatiotemporal traits of spontaneous activity, including the layer-specific interhemispheric correlation pattern. Additionally, the highly variable time delays between bilateral neuroelectric pairs suggested the involvement of multiple neural signal pathways for each spontaneous neural event. Considered with the rsfMRI results, this work demonstrated that substantial, immediate neural correlates underpin the interhemispheric functional connectivity which was found with sparsely sampled rsfMRI.

Acknowledgments The authors thank Drs. Jitendra Sharma and Robert Haslinger for their valuable comments on this manuscript. This work was supported by grants from the National Institutes of Health (Grant Number 5R01EB002066, R01 EB001954) and a Korea Science and Engineering Foundation (KOSEF) grant that was funded by the Korean government (Grant number NRF-2006-2005399, M10644000028-06N4400-02810).

\section{References}

Bannister AP (2005) Inter-and intra-laminar connections of pyramidal cells in the neocortex. Neurosci Res 53(2):95-103

Biswal B, Yetkin F, Haughton V, Hyde J (1995) Functional connectivity in the motor cortex of resting human brain using echo-planar MRI. Magn Reson Med 34(4):537-541

Buxton RB, Uludağ K, Dubowitz DJ, Liu TT (2004) Modeling the hemodynamic response to brain activation. Neuroimage 23:S220-S233

Chapman C, Trepel C, Ivanco T, Froc D, Wilson K, Racine R (1998) Changes in field potentials and membrane currents in rat sensorimotor cortex following repeated tetanization of the corpus callosum in vivo. Cereb Cortex 8(8):730

Crochet S, Petersen CCH (2009) Cortical dynamics by layers. Neuron 64(3):298-300

Csercsa R, Dombovari B, Fabo D, Wittner L, Erss L, Entz L, Solyom A, Rasonyi G, Szcs A, Kelemen A (2010) Laminar analysis of slow wave activity in humans. Brain 133(9):2814

Douglas RJ, Martin KAC (2004) Neuronal circuits of the neocortex. Annu Rev Neurosci 27:419-451

Einevoll G, Pettersen K, Devor A, Ulbert I, Halgren E, Dale A (2007) Laminar population analysis: estimating firing rates and evoked synaptic activity from multielectrode recordings in rat barrel cortex. J Neurophysiol 97(3):2174

Fox M, Raichle M (2007) Spontaneous fluctuations in brain activity observed with functional magnetic resonance imaging. Nat Rev Neurosci 8(9):700-711

Fox M, Snyder A, Zacks J, Raichle M (2005) Coherent spontaneous activity accounts for trial-to-trial variability in human evoked brain responses. Nat Neurosci 9(1):23-25
Friston K (1994) Functional and effective connectivity in neuroimaging: a synthesis. Hum Brain Mapp 2(1-2):56-78

Goense J, Merkle H, Logothetis NK (2012) High-resolution fMRI reveals laminar differences in neurovascular coupling between positive and negative BOLD responses. Neuron 76(3):629-639

Harel N, Lin J, Moeller S, Ugurbil K, Yacoub E (2006) Combined imaging-histological study of cortical laminar specificity of fMRI signals. Neuroimage 29(3):879-887

Haslinger R, Ulbert I, Moore CI, Brown EN, Devor A (2006) Analysis of LFP phase predicts sensory response of barrel cortex. J Neurophysiol 96(3):1658

Hayama T, Ogawa H (1997) Regional differences of callosal connections in the granular zones of the primary somatosensory cortex in rats. Brain Res Bull 43(3):341-347

Hyvarinen A, Oja E (1997) A fast fixed-point algorithm for independent component analysis. Neural Comput 9(7):1483-1492

Isseroff A, Schwartz M, Dekker J, Goldman-Rakic P (1984) Columnar organization of callosal and associational projections from rat frontal cortex. Brain Res 293(2):213-223

Johnston J, Vaishnavi S, Smyth M, Zhang D, He B, Zempel J, Shimony J, Snyder A, Raichle M (2008) Loss of resting interhemispheric functional connectivity after complete section of the corpus callosum. J Neurosci 28(25):6453

Kim YR, Biswal BB, Rosen BR (2008) Comparison of spontaneous electrophysiological and fMRI fluctuations during rest in rat brain cortex. In: 16th Annual Meeting of International Society of Magnetic Resonance in Medicine, Toronto, Canada, 2008. Toronto, Canada

Kiviniemi V, Kantola J-H, Jauhiainen J, Hyvärinen A, Tervonen O (2003) Independent component analysis of nondeterministic fMRI signal sources. Neuroimage 19(2):253-260

Lefort S, Tomm C, Floyd Sarria J, Petersen CCH (2009) The excitatory neuronal network of the $\mathrm{C} 2$ barrel column in mouse primary somatosensory cortex. Neuron 61(2):301

Leopold DA, Maier A (2012) Ongoing physiological processes in the cerebral cortex. Neuroimage 62(4):2190-2200

Leopold DA, Murayama Y, Logothetis NK (2003) Very slow activity fluctuations in monkey visual cortex: implications for functional brain imaging. Cereb Cortex 13(4):422-433

Liu X, Zhu X-H, Zhang Y, Chen W (2011) Neural origin of spontaneous hemodynamic fluctuations in rats under BurstSuppression anesthesia condition. Cereb Cortex 21(2):374-384

Lowe M, Mock B, Sorenson J (1998) Functional connectivity in single and multislice echoplanar imaging using resting-state fluctuations. Neuroimage 7(2):119-132

Lu H, Zuo Y, Gu H, Waltz JA, Zhan W, Scholl CA, Rea W, Yang Y, Stein EA (2007) Synchronized delta oscillations correlate with the resting-state functional MRI signal. Proc Natl Acad Sci 104(46): 18265

Lubke J, Feldmeyer D (2007) Excitatory signal flow and connectivity in a cortical column: focus on barrel cortex. Brain Struct Funct 212(1):3-17

Lumer ED, Edelman GM, Tononi G (1997) Neural dynamics in a model of the thalamocortical system. II. The role of neural synchrony tested through perturbations of spike timing. Cereb Cortex 7(3):228-236

Magnuson ME, Thompson GJ, Pan W-J, Keilholz SD (2014) Effects of severing the corpus callosum on electrical and BOLD functional connectivity and spontaneous dynamic activity in the rat brain. Brain Connectivity 4(1):15-29

Magri C, Schridde U, Murayama Y, Panzeri S, Logothetis NK (2012) The amplitude and timing of the BOLD signal reflects the relationship between local field potential power at different frequencies. J Neurosci 32(4):1395-1407 
Maier A, Adams GK, Aura C, Leopold DA (2010) Distinct superficial and deep laminar domains of activity in the visual cortex during rest and stimulation. Front Syst Neurosci 4:31

Majeed W, Magnuson M, Keilholz SD (2009) Spatiotemporal dynamics of low frequency fluctuations in BOLD fMRI of the rat. J Magn Reson Imaging 30(2):384-393

Mandeville JB, Jenkins BG, Kosofsky BE, Moskowitz MA, Rosen BR, Marota JJA (2001) Regional sensitivity and coupling of BOLD and CBV changes during stimulation of rat brain. Magn Reson Med 45(3):443-447

Mohajerani M, McVea D, Fingas M, Murphy T (2010) Mirrored bilateral slow-wave cortical activity within local circuits revealed by fast bihemispheric voltage-sensitive dye imaging in anesthetized and awake mice. J Neurosci 30(10):3745

Nir Y, Fisch L, Mukamel R, Gelbard-Sagiv H, Arieli A, Fried I, Malach R (2007) Coupling between neuronal firing rate, gamma LFP, and BOLD fMRI is related to interneuronal correlations. Curr Biol 17(15):1275-1285

Nir Y, Mukamel R, Dinstein I, Privman E, Harel M, Fisch L, GelbardSagiv H, Kipervasser S, Andelman F, Neufeld MY (2008) Interhemispheric correlations of slow spontaneous neuronal fluctuations revealed in human sensory cortex. Nat Neurosci 11(9):1100-1108

Pan W-J, Thompson G, Magnuson M, Majeed W, Jaeger D, Keilholz S (2010) Simultaneous FMRI and electrophysiology in the rodent brain. J Vis Exp 42:1901

Pan W-J, Thompson G, Magnuson M, Majeed W, Jaeger D, Keilholz S (2011) Broadband local field potentials correlate with spontaneous fluctuations in functional magnetic resonance imaging signals in the rat somatosensory cortex under isoflurane anesthesia. Brain Connectivity 1(2):119-131

Pan W-J, Thompson GJ, Magnuson ME, Jaeger D, Keilholz S (2013) Infraslow LFP correlates to resting-state fMRI BOLD signals. Neuroimage 74:288-297

Peeters R, Tindemans I, De Schutter E, Van der Linden A (2001) Comparing BOLD fMRI signal changes in the awake and anesthetized rat during electrical forepaw stimulation. Magn Reson Imaging 19(6):821-826

Quigley M, Cordes D, Turski P, Moritz C, Haughton V, Seth R, Meyerand M (2003) Role of the corpus callosum in functional connectivity. Am J Neuroradiol 24(2):208

Raichle M, Mintun M (2006) Brain work and brain imaging. Annu Rev Neurosci 29:449-476

Scholvinck ML, Maier A, Ye FQ, Duyn JH, Leopold DA (2010) Neural basis of global resting-state fMRI activity. Proc Natl Acad Sci 107(22):10238
Seggie J, Berry M (1972) Ontogeny of interhemispheric evoked potentials in the rat: significance of myelination of the corpus callosum. Exp Neurol 35(2):215-232

Shmuel A, Leopold DA (2008) Neuronal correlates of spontaneous fluctuations in fMRI signals in monkey visual cortex: implications for functional connectivity at rest. Hum Brain Mapp 29(7):751-761

Shmueli K, van Gelderen P, de Zwart JA, Horovitz SG, Fukunaga M, Jansma JM, Duyn JH (2007) Low frequency fluctuations in the cardiac rate as a source of variance in the resting-state fMRI BOLD signal. Neuroimage 38(2):306

Shulman R, Rothman D, Behar K, Hyder F (2004) Energetic basis of brain activity: implications for neuroimaging. Trends Neurosci 27(8):489-495

Silva AC, Koretsky AP (2002) Laminar specificity of functional MRI onset times during somatosensory stimulation in rat. Proc Natl Acad Sci 99(23): 15182

Silva AC, Koretsky AP, Duyn JH (2007) Functional MRI impulse response for BOLD and $\mathrm{CBV}$ contrast in rat somatosensory cortex. Magn Reson Med 57(23):1110-1118

Thompson GJ, Merritt MD, Pan W-J, Magnuson ME, Grooms JK, Jaeger D, Keilholz SD (2013) Neural correlates of time-varying functional connectivity in the rat. Neuroimage 83:826-836

Tian P, Teng IC, May LD, Kurz R, Lu K, Scadeng M, Hillman E, De Crespigny AJ, D'Arceuil HE, Mandeville JB (2010) Cortical depth-specific microvascular dilation underlies laminar differences in blood oxygenation level-dependent functional MRI signal. Proc Natl Acad Sci 107(34):15246

Uddin LQ, Mooshagian E, Zaidel E, Scheres A, Margulies DS, Kelly AC, Shehzad Z, Adelstein JS, Castellanos FX, Biswal BB (2008) Residual functional connectivity in the split-brain revealed with resting-state fMRI. Neuroreport 19(7):703

Vizuete J, Pillay S, Ropella K, Hudetz A (2014) Graded defragmentation of cortical neuronal firing during recovery of consciousness in rats. Neuroscience 275:340-351

Williams KA, Magnuson M, Majeed W, LaConte SM, Peltier SJ, Hu X, Keilholz SD (2010) Comparison of $\alpha$-chloralose, medetomidine and isoflurane anesthesia for functional connectivity mapping in the rat. Magn Reson Imaging 28(7):995-1003

Wise SP (1975) The laminar organization of certain afferent and efferent fiber systems in the rat somatosensory cortex. Brain Res 90(1):139 